\title{
EVOLUÇÃO DA CARDIOPATIA CHAGÁSICA CRONICA HUMANA NO SERTÃO DO ESTADO DA PARAÍBA, BRASIL, NO PERÍODO DE 4,5 ANOS.
}

\author{
José Borges Pereira, Rivaldo Venâncio da Cunha, \\ Henry P. Faraco Willcox e José Rodrigues Coura.
}

\begin{abstract}
Foram desenvolvidos dois estudos seccionais sobre a doença de Chagas crônica, com intervalo de 4,5 anos, envolvendo as populaçôes urbanas dos municipios de Água Branca, Catingueira, Emas, Imaculada, Mãe D'Agua, Olho D'Agua, Piancó e São José de Caiana, situados na regiāo do Sertão do Estado da Paraíba. A evolufão da cardiopatia foi avaliada em um grupo de 125 pares de pacientes chagásicos crônicos e não-chagásicos do mesmo sexo, idade e município de origem, através do exame eletrocardiográfico (ECG) de repouso. Foram considerados os seguintes tipos de evolução: inalterada-quando não havia mudança no padrão inicial do ECG; progressiva - quando havia mudança no padrão do ECG de normal para alterado ou pelo agravamento das alterações e normalização do ECG. No grupo de chagásicos a evolução inalterada ocorreu em $101(80,8 \%)$ pacientes, progressiva em $13(10,4 \%)$ e normalização do ECG em $11(8,8 \%)$, enquanto no grupo de não-chagásicos foram observadas as mencionadas evolucões respectivamente em 117 (93,6\%), 6 (4,8\%) e 2 (1,6\%) pacientes. Com esses dados podemos afirmar que a proporção de participação do componente etiologico exclusivamente chagásico na progressão da cardiopatia chagásica crônica foi de 5,9\%, estimando-se uma média anual de 1,3\%. Não houve diferença significativa nas freqüências de evolucão progressiva em relação ao sexo dos pacientes, tanto no grupo de chagásicos como no de não-chagásicos. Por outro lado, a progressão da cardiopatia ocorreu mais precocemente nos chagásicos. A letalidade por cardiopatia foi $1,6 \%$ (2 casos) no grupo de chagásicos e de zero no de não chagásicos, no periodo considerado. Esses dados sobre a morbimortalidade podem ser considerados significativamente inferiores aos encontrados em áreas endêmicas como Virgem da Lapa e Pains-Iguatama, em Minas Gerais, provavelmente expressando o menor poder patogênico da infecção humana pelo Trypanosoma cruzi no Sertâo da Paraíba.
\end{abstract}

Palavras-chaves: Doença de Chagas. Estudo longitudinal. Cardiopatia chagásica crônica. Estado da Paraiba.

Os estudos longitudinais sobre a morbidade da doença de Chagas crônica têm mostrado que, nessa fase da infecção, a cardiopatia evolui progressivamente em alguns pacientes, enquanto em outros mantém-se inalterada ao longo dos anos. Além disso, pacientes nessa fase crônica podem permanecer sem quaisquer manifestações cardiovascular e/ou digestiva, classificados na forma clínica indeterminada, possivelmente por toda a vida, conforme trabalhos de Dias 5 em Bambuí, Minas Gerais, e de outros autores ${ }^{11} 12$ em outras áreas endèmicas do Brasil.

Essas diferenças no comportamento evolutivo da doença de Chagas crônica têm suscitado questões em torno da definição sobre que pacientes apresentam

Instituto Oswaldo Cruz/FIOCRUZ, Rio de Janeiro, RJ, Brasil. Endereço para correspondência: Dr. José Borges Pereira. Depto. de Medicina Tropical/FIOCRUZ, CP: 926- 21040 Rio de Janeiro, RJ. Recebido para publicaçăo em 10/01/90. potencial evolutivo, capaz de levá-los à evolução da forma clínica indeterminada para a forma cardíaca e/ou digestiva (megas) em um determinado momento da vida. A resposta, quando e se definida, traria um significativo avanço no conhecimento da história natural da infecção humana pelo Trypanosoma cruzi.

Estudos epidemiológicos descritivos assinalam diferenças quanto ao poder evolutivo da doença de Chagas em função de variáveis como: áreas endêmicas 1478 , característica clínica da fase aguda 5 , grupos etários dos pacientes crônicos 412 e outras.

Tomando por base a diferença regional que envolve a morbidade da doença de Chagas, confirmada por nós ao compararmos os resultados das freqüências de cardiopatia e esofagopatia encontradas no estudo seccional realizado no Sertão da Paraiba ${ }^{11}$ e os resultados obtidos nas áreas de Virgem da Lapa e Pains-Iguatama em Minas Gerais, é que promovemos o presente estudo longitudinal nessa região do Estado da Paraiba. 


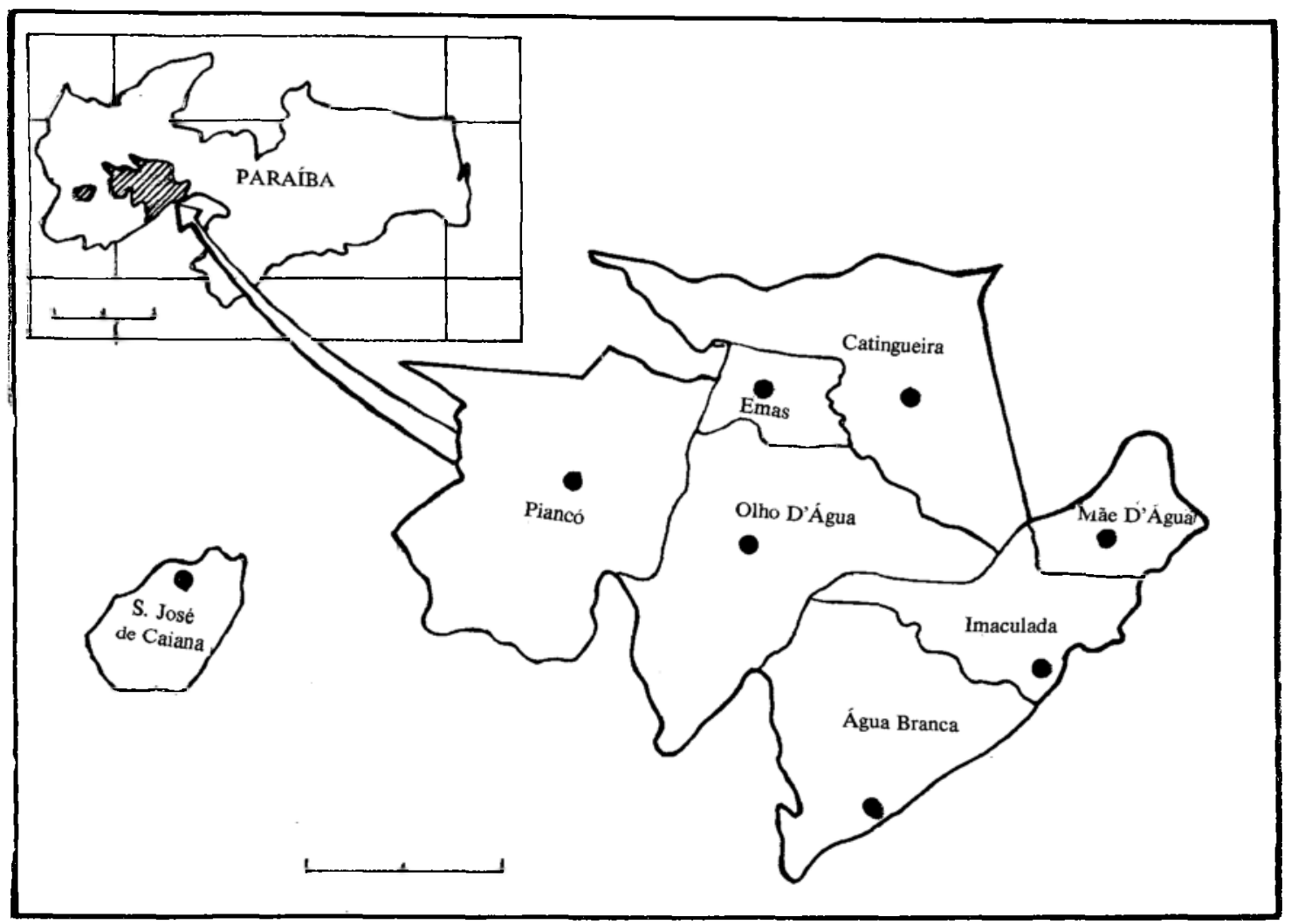

Figura 1 - Estado da Paraíba - Localização da área estudada.

\section{MATERIAL E MÉTODOS}

Em janeiro de 1985 foi realizado o primeiro estudo seccional sobre a morbidade da doença de Chagas crônica humana, envolvendo populações urbanas dos municípios de Água Branca, Catingueira, Emas, Imaculada, Mãe D'Água, Olho D’Água, Piancó e São José de Caiana (Figura 1). Nessa ocasião buscamos definir a freqüência da cardiopatia chagásica crônica em uma amostra de 305 pares de pacientes chagásicos e não-chagásicos do mesmo sexo, idade e município de origem.

Em julho de 1989, 4,5 anos depois, foi realizado o segundo estudo seccional, que se constitui no atual estudo evolutivo, no qual procuramos observar o comportamento da cardiopatia e da mortalidade a ela atribuivel nos grupos de pacientes chagásicos e nãochagásicos estabelecidos no primeiro exame.

Da amostra inicial de 610 pacientes, conseguimos reexaminar 323 (53\%); emigraram 127 (21\%), não compareceram ao exame $57(9,3 \%)$, não foram convocados em tempo $95(15,4 \%)$ e morreram
$8(1,3 \%)$. Do grupo de reexaminados foi possivel rever 125 pares de acordo com os critérios adotados no primeiro estudo seccional. Esses 125 pares compõem a amostra do presente trabalho, a qual está composta por 89 pares de mulheres e 36 pares de homens com idades, à época do primeiro exame, variando de 4 a 77 anos (média de 39,6 $\pm 15,6$ ). Tratando-se de um estudo do tipo caso-controle, as defecções, embora reduzindo o número e pares, não alteram a análise evolutiva da casuística restante por serem os mesmos pares.

Todos os pacientes toram submetidos, em ambos os estudos, ao exame eletrocardiográfico $\mathrm{em}$ repouso, obtendo-se o registro das doze derivações clássicas com D2 longo em caso de arritmias. A leitura e a interpretação dos traçados obedeceram aos critérios da NYHA (New York Heart Association) ${ }^{10}$, considerando normais as freqüências de 60 a 120 ciclos por minuto.

Classificamos a evolução eletrocardiográfica, representando a evolução da cardiopatia, em três tipos: inalterada - quando não havia mudança no 
padrão inicial do ECG; progressiva - quando havia mudança do padrão inicial do ECG: de normal para alterado ou com o agravamento das alterações e a normalização do ECG.

Em todos os pacientes reexaminados foi confirmada a soropositividade ou a soronegatividade detectada no primeiro exame, através dos testes de imunofluorescência indireta e de fixação do complemento. Não foram realizados os exames radiográficos do coração e do esôfago no presente estudo.

\section{RESULTADOS}

Entre os 125 pares considerados $(40 \%$ da amostra inicial), por ocasião do primeiro exame havia no grupo de chagásicos 92 pacientes $(73,6 \%)$ com ECG normais e $33(26,4 \%)$ com ECG alterados, enquanto no grupo de não-chagásicos havia, respectivamente, $115(92,0 \%)$ e $10(8,0 \%)$ pacientes.

A análise evolutiva dos ECG revelou no grupo de chagásicos 12 casos novos $(13,0 \%)$ de ECG alterados (nos 1 a 12, Tabela 4) e no grupo de nãochagásicos 6 casos novos $(5,2 \%)$ (Tabela 5), caracterizando assim a incidência de cardiopatia 2,5 vezes maior no grupo de chagásicos. Entre os pacientes que apresentavam ECG alterados no primeiro exame, verificou-se o agravamento em um caso no grupo de chagásicos (no 13, Tabela 4) e nenhum caso no grupo de não-chagásicos. Considerando ainda esses pacientes, assinalamos a normalização do traçado (Tabela 6) em 11 casos chagásicos $(33,3 \%)$ e em 2 casos nãochagásicos $(20,0 \%)$, expressando assim o maior poder de mutabilidade eletrocardiográfica nos pacientes com cardiopatia chagásica em relação àqueles com cardiopatia de outra etiologia.

$\mathrm{Na}$ Tabela 1 está assinalada a evolução eletrocardiográfica global nos 125 pares. O emprego do método de Sheps 15 permite-nos estimar a proporção de participação do componente etiológico exclusiva- mente chagásico na evolução progressiva em 5,9\% com uma média anual de progressão de $1,3 \%$ dos casos.

$\mathrm{Na}$ Tabela 2 estão assinalados os percentuais da evolução eletrocardiográfica progressiva nos 125 pares de acordo com o grupo etário.

Na Tabela 3 está asssinạlada a evolução eletrocardiográfica progressiva no grupo de pacientes chagásicos de acordo com o sexo e o grupo etário.

As Tabelas 4 e 5 mostram, respectivamente, as caracteristicas dos pacientes chagásicos e dos nãochagásicos que evoluiram progressivamente. Deve-se ressaltar o maior número de casos que evoluiram com o aparecimento de alteração primária da repolarização ventricular, somente um caso com extra-sístole ventricular isolada (n.o 13, Tabela 4) e alteração característica da cardiopatia chagásica crônica somente nos casos nos 4 e 9 , Tabela 4.

A Tabela 7 mostra as caracteristicas dos pacientes que evoluíram para o óbito. São 4 casos entre os chagásicos (soropositivos) e 4 entre os não-chagásicos (soronegativos). Em somente dois casos (n.s 7 e 8)

Tabela 1-Evolução eletrocardiográfica(*) em 125 pares de pacientes chagásicos e não-chagásicos, no período de 4,5 anos.

\begin{tabular}{lrrrc}
\hline \multirow{2}{*}{ Evolução } & \multicolumn{2}{c}{ Chagásicos } & \multicolumn{2}{c}{ Não-chagásicos } \\
& No & \multicolumn{1}{c}{$\%$} & \multicolumn{1}{c}{ N. } & \multicolumn{1}{c}{$\%$} \\
\hline Inalterada & 101 & 80,8 & 117 & 93,6 \\
Progressiva & 13 & 10,4 & 6 & $4,8^{(* *)}$ \\
Normalização do ECG & 11 & 8,8 & 2 & 1,6 \\
\hline Total & 125 & 100,0 & 125 & 100,0 \\
\hline
\end{tabular}

(*) Considerando todas as alteraçōes.

$\left({ }^{* *}\right)$ Não significativa, de acordo com o teste do Qui-quadrado para $\alpha \gtrless 0,05$.

Tabela 2-Evolução eletrocardiográfica(*) progressiva em 125 pares de chagásicos e não-chagásicos de acordo com o, grupo etário.

\begin{tabular}{ccccccc}
\hline $\begin{array}{c}\text { Grupo } \\
\text { etário }\end{array}$ & $\begin{array}{c}\text { Exami- } \\
\text { nados }\end{array}$ & $\begin{array}{c}\text { Chagásicos } \\
\text { C/evolu. } \\
\text { progres. }\end{array}$ & $\%$ & $\begin{array}{c}\text { Exami- } \\
\text { nados }\end{array}$ & $\begin{array}{c}\text { Não-chagásicos } \\
\text { C/evolu. } \\
\text { progres. }\end{array}$ & $\%$ \\
\hline $4-19$ & 17 & 0 & - & 17 & 0 & - \\
$20-39$ & 32 & 3 & 9,4 & 32 & 1 & 3,1 \\
$40-59$ & 57 & 9 & 15,8 & 57 & 3 & 5,3 \\
$\geq 60$ & 19 & 1 & 5,3 & 19 & 2 & 10,5 \\
\hline Total & 125 & 13 & 10,4 & 125 & 6 & 4,8 \\
\hline
\end{tabular}

(*) Considerando todas as alteraçōes. 
Pereira JB, Cunha RV, Willcox HPF, Coura JR. Evolução da cardiopatia chagásica crónica humana no Sertão do Estado da Paraíba, Brasil, no periodo de 4,5 anos. Revista da Sociedade Brasileira de Medicina Tropical 23: 141-147, jul-set, 1990.

Tabela 3-Evolução eletrocardiográfica(*) progressiva em 125 chagásicos, de acordo com o sexo e grupo etário.

\begin{tabular}{|c|c|c|c|c|c|c|}
\hline \multirow{2}{*}{$\begin{array}{l}\text { Grupo } \\
\text { etário }\end{array}$} & \multicolumn{3}{|c|}{ Homens } & \multicolumn{3}{|c|}{ Mulheres } \\
\hline & $\begin{array}{c}\text { Exami- } \\
\text { nados }\end{array}$ & $\begin{array}{l}\text { C/evolu. } \\
\text { progres. }\end{array}$ & $\%$ & $\begin{array}{c}\text { Exami- } \\
\text { nados }\end{array}$ & $\begin{array}{l}\mathrm{C} / \text { evolu. } \\
\text { progres. }\end{array}$ & $\%$ \\
\hline $4-19$ & 7 & 0 & - & 10 & 0 & - \\
\hline $20-39$ & 8 & 2 & 25,0 & 24 & 1 & 4,2 \\
\hline $40-59$ & 15 & 3 & 20,0 & 42 & 6 & 14,3 \\
\hline$\geq 60$ & 6 & 0 & - & 13 & 1 & 7,7 \\
\hline Total & 36 & 5 & $13,9^{(* *)}$ & 89 & 8 & $9,0^{(* *)}$ \\
\hline
\end{tabular}

(*) Considerando todas as alterações.

(**) Não significativa, de acordo com o teste da diferença das proporções para $\alpha \overline{<} 0,05$.

Tabela 4-Pacientes chagásicos que apresentaram evolução progressiva.

\begin{tabular}{|c|c|c|c|c|c|}
\hline No & Registro & Idade & Sexo & ECG em jan/85 & ECG em jul/89 \\
\hline 1 & 198 & 40 & $f$ & Normal & APRV \\
\hline 2 & 226 & 47 & f & ", & TS \\
\hline 3 & 425 & 48 & $\mathrm{~m}$ & $"$ & APRV \\
\hline 4 & 472 & 38 & $\mathrm{~m}$ & $"$ & BRD III + HBAE \\
\hline 5 & 553 & 47 & f & $"$ & TS \\
\hline 6 & 598 & 60 & $\mathbf{f}$ & $"$ & TS \\
\hline 7 & 602 & 49 & f & " & APRV \\
\hline 8 & 627 & 40 & f & $"$ & BRD I \\
\hline 9 & 636 & 53 & f & $"$ & BRD III \\
\hline 10 & 646 & 23 & $\mathrm{~m}$ & $"$ & APRV \\
\hline 11 & 651 & 43 & $\mathrm{~m}$ & " & APRV \\
\hline 12 & 676 & 53 & $\mathbf{m}$ & " & APRV \\
\hline 13 & 532 & 31 & $\mathrm{f}$ & BRD III & $\mathrm{BRD}$ III + HBAE + EV \\
\hline
\end{tabular}

APRV = alteração primária da repolarizaçăo ventricular, $T S=$ taquicardia sinusal; $B R D=$ bloqueio do ramo direito; $\mathrm{HBAE}=$ hemibloqueio anterior esquerdo; $\mathrm{EV}=$ extra-sistoles ventriculares.

Tabela 5-Pacientes não-chagásicos que apresentaram evolução progressiva.

\begin{tabular}{|c|c|c|c|c|c|}
\hline No & Registro & Idade & Sexo & ECG em jan/85 & ECG em jul/89 \\
\hline 1 & 354 & 73 & $\mathrm{~m}$ & Normal & APRV \\
\hline 2 & 607 & 62 & f & " & TS \\
\hline 3 & 608 & 22 & f & $"$ & HBAE \\
\hline 4 & 628 & 57 & f & $"$ & APRV \\
\hline 5 & 667 & 43 & f & ” & APRV \\
\hline 6 & 691 & 46 & f & $"$ & APRV \\
\hline
\end{tabular}

podemos atribuir responsabilidade pelo óbito à cardiopatia, identificando uma letalidade de $1,6 \%$ entre os chagásicos e de zero entre os não-chagásicos.

\section{DISCUSSÃO}

Por ocasião do primeiro estudo seccional sobre a morbidade da doença de Chagas nessa região do Sertão da Paraiba ${ }^{11}$, assinalamos freqüências de cardiopatia e de esofagopatia chagásicas significativamente inferiores às registradas em outras áreas endêmicas: Virgem da Lapa e Pains-Iguatama-Minas Gerais $^{4}{ }^{12}$, Mambai-Goiás ${ }^{3}$ e São Felipe-Bahia 7 , para o que não dispúnhamos de explicações consistentes, mas ressaltávamos que, provavelmente, se devia a menor patogenicidade na relação homem $-T$. cruzi nessa região do Estado da Paraíba.

Confirmando esses resultados do estudo transversal, o atual estudo longitudinal revela um baixo poder evolutivo da doença de Chagas cardíacà crônica no Sertão da Paraíba em relação ao encontrado nas áreas citadas acima dos Estados de Minas Gerais e Bahia, tendo em vista que o percentual de evolução progressiva da cardiopatia chagásica crônica no Sertão da Paraíba pode ser estimado em 1,3\% dos casos por ano, enquanto que nas demais áreas citadas esta proporção oscila entre $2,7 \%$ em Pains-Iguatama e 
Pereira JB, Cunha RV, Willcox HPF, Coura JR. Evolução da cardiopatia chagásica crônica humana no Sertão do Estado da Paraíba, Brasil, no periodo de 4,5 anos. Revista da Sociedade Brasileira de Medicina Tropical 23: 141-147, jul-set, 1990.

Tabela 6-Pacientes que apresentaram normalizaçâo do ECG.

\begin{tabular}{|c|c|c|c|c|c|c|}
\hline No & Registro & Idade & Sexo & Sorologia & ECG em jan/85 & ECG em jul/89 \\
\hline 1 & 171 & 30 & f & Negativa & APRV & Normal \\
\hline 2 & 623 & 44 & f & $"$ & $\mathrm{EVi}$ & $"$ \\
\hline 3 & 27 & 30 & $\mathrm{f}$ & Positiva & BV - QRS & $"$ \\
\hline 4 & 32 & 38 & $\mathrm{f}$ & "’ & APRV & " \\
\hline 5 & 151 & 30 & $f$ & ” & APRV & $"$ \\
\hline 6 & 251 & 30 & $\mathrm{f}$ & " & APRV & $"$ \\
\hline 7 & 254 & 44 & $\mathrm{~m}$ & " & BAV I & $"$ \\
\hline 8 & 304 & 43 & $\mathrm{f}$ & $"$ & BAV I & $"$ \\
\hline 9 & 326 & 38 & m & $"$ & TS & $"$ \\
\hline 10 & 351 & 59 & $\mathrm{f}$ & $"$ & $\mathrm{EVi}$ & " \\
\hline 11 & 353 & 15 & $\mathrm{f}$ & $"$ & $\mathrm{EVi}$ & $"$ \\
\hline 12 & 582 & 65 & $\mathrm{f}$ & $"$ & ESV & $"$ \\
\hline 13 & 695 & 45 & $f$ & $"$ & BAV I & $"$ \\
\hline
\end{tabular}

$\mathrm{EVj}=$ extra-sistole ventricular isolada; $\mathrm{BV}-\mathrm{QRS}=$ baixa voltagem do QRS; BAVI = bloqueio aurículo-ventricular do $19 \mathrm{grau} ; \mathrm{ESV}=$ extrasistole supraventricular.

Tabela 7-Pacientes que evoluíram para o bbito no periodo de 4,5 anos.

\begin{tabular}{cccccll}
\hline No & Registro & Idade & Sexo & Sorologia & ECG em jan/85 & Causa da morte* \\
\hline 1 & 25 & 34 & $\mathrm{f}$ & Negativa & Normal & Anemia aguda** \\
2 & 334 & 56 & $\mathrm{~m}$ &, & Normal & Anemia aguda*** \\
3 & 355 & 54 & $\mathrm{f}$ &, & Normal & Acidente vascular cerebral \\
4 & 594 & 66 & $\mathrm{f}$ & $"$ & Normal & Ignorada \\
5 & 282 & 23 & $\mathrm{~m}$ & Positiva & Normal & Anemia aguda*** \\
6 & 364 & 38 & $\mathrm{~m}$ &, & Normal & Anemia aguda*** \\
7 & 648 & 63 & $\mathrm{~m}$ & $"$ & BRD III, HBAE & Edema agudo de pulmāo \\
8 & 650 & 40 & $\mathrm{f}$ &, & BRD III, HBAE, EV & Parada cardiorrespiratória \\
\hline
\end{tabular}

* Dados obtidos nos cartórios da região.

** Durante o trabalho de parto.

**** Em conseqüência de violência.

$5,2 \%$ em Virgem da Lapa. Do mesmo modo, é significativamente menor a letalidade por cardiopatia chagásica, $0,35 \%$ ao ano na região do presente estudo, em comparação com $1,70 \%$ de Pains-Iguatama e $1,45 \%$ de Virgem da Lapa.

Outro achado, que destacamos na análise comparativa, é o de que no grupo de pacientes chagásicos do Sertão da Paraíba, tanto no estudo seccional quanto no longitudinal, há um número significativamente inferior de arritmias graves como extra-sístoles ventriculares polimórficas ou bigeminadas ou em salvas, em confronto com as elevadas freqüências encontradas nos estudos realizados nas outras áreas mencionadas no texto. Esta observação provavelmente seja mais um fator que expressa o menor grau de lesão cardíaca existente nos pacientes chagásicos dessa região paraibana.

A análise comparativa do risco de progressão da cardiopatia chagásica crônica em áreas endêmicas do
Brasil, nas quais houve estudos com grupos-controles não-chagásicos, mostra riscos de 6,4 vezes em PainsIguatama, de 5 vezes em Virgem da Lapa 2,2 vezes no presente trabalho e de 1,2 vezes em áreas do sul do Rio Grande do Sull. Embora o valor encontrado no Rio Grande do Sul seja menor que o do Sertão da Paraíba, há maior ocorrência de arritmias de risco nos chagásicos daquela região, caracterizando assim maior gravidade da cardiopatia chagásica crônica em relação ao Sertão do Estado da Paraiba.

Fora do Brasil, especificamente na Venezuela, em Belén ${ }^{14}$ e Carabobo ${ }^{9}$, foram desenvolvidos estudos longitudinais sobre a cardiopatia chagásica crônica que revelaram potenciais de evolução progressiva também superiores ao encontrado no Sertão da Paraíba. Ressalta-se que o estudo em Carabobo não utilizou grupo-controle não-chagásico.

Tanto no presente trabalho como em outros realizados em diferentes áreas endêmicas 4 12, tem 
sido observada a normalização do ECG mais freqüente no grupo de chagásicos, caracterizando a mutabilidade eletrocardiográfica que provavelmente expressa a maior dinâmica eletrofisiológica nos corações desses pacientes, indicando para nós muito mais um processo de reorganização evolutiva no sentido progressivo do que regressivo, em acordo com Brasil ${ }^{2}$.

Os índices de letalidade devido à doença de Chagas têm sido tanto maiores quanto maiores sao os indicadores de morbidade cardíaca nas áreas estudadas 312 . Assim é que consideramos baixo o índice de $1,6 \%$ encontrado no Sertão da Paraíba em relação aos de $17,0 \%$ (em dez anos) e de $8,9 \%$ (em seis anos), respectivamente, em Pains-Iguatama e Virgem da Lapa, Minas Gerais.

Os dois pacientes que evoluíram para o óbito atribuivel à cardiopatia chagásica (nos 7 e 8 da Tabela 6) apresentavam alterações consideradas de alto risco nos trabalhos de Prata ${ }^{13}$ e de Dias e Kloetzel6. É importante assinalar que o paciente mais jovem morreu subitamente, enquanto o mais idoso desenvolveu o estado clínico de insuficiência cardiaca congestiva, chegando ao óbito por edema agudo de pulmão. Por outro lado, não foi assinalado nenhum caso de óbito por cardiopatia chagásica em pacientes que no primeiro exame tinham ECG normal.

Diante dos resultados apresentados no presente trabalho e de outros trabalhos referidos no texto, podemos afirmar que a evolução da doença de Chagas, na sua apresentação cardíaca, na região do Sertão da Paraiba, apresenta-se com baixos índices de morbidade e letalidade, provavelmente em conseqüência do menor poder patogênico resultante da relação homemT. cruzi, caracterizando importante diferença regional que exige investigações epidemiológicas e parasitológicas capazes de èsclarecê-la.

\section{SUMMARY}

Two sectional studies about chronic Chagas' disease were performed at a 4.5 year interval, involving the urban populations of Água Branca, Catingueira, Emas, Imaculada, Mãe D'Água, Olho D'Água, Piancó and Sâo José de Caiana counties in the Sertão region of the State of Paraiba. The evolution of heart disease was evaluated in 125 matched pairs of chronic chagasic and non-chagasic patients of the same sex, age and county of origin through electrocardiograms (ECG) at rest. The following evaluation criteria were considered: unchanged - no change in the original ECG pattern; progressive-changes in ECG pattern from normal to abnormal or deterioration of ECG abnormalities; and ECG normalization. In chagasic patients evolution of the disease was unchanged in 101 (80.8\%), progressive in $13(10.4 \%)$ and ECG normalization in 11 (8.8\%), while those observed in non-chagasic patients were respectively values $117(93.6 \%), 6(4.8 \%)$ and $2 \%)$ patients. Findings indicate that the share of Chagas-linked etiological component affecting the development of chronic Chagas cardiopathy was $5.9 \%$ with an estimated annual average of $1.3 \%$. There was no significant difference in the frequency of progressive disease between the sexes either in the chagasic or in the non-chagasic group. On the other hand, progression of heart disease occurred earlier among chagasic patients. Lethality caused by heart disease was $1.6 \%$ (2 cases) in the chagasic group and zero in the nonchagasic group during the period under survey. These morbidity and mortality rates were significantly lower than those found in endemic areas such as Virgem da Lapa and Pains-Iguatama in the State of Minas Gerais and can probably be attibuted to the weaker pathogenic it of human infection by Trypanosoma cruzi in the Paraiba hinterland

Key-words: Chagas' disease. Longitudinal study. Chronic Chagas cardiopathy. State of Paraiba.

\section{AGRADECIMENTOS}

Aos técnicos Mozar José Coelho e Joaquim Mendes pelo incansável apoio na mobilização da população. Aos funcionários dos Centros de Saúde dos municípios estudados, pela ajuda na preparação dos pacientes para os exames. Ao Dr. Nelson Chagas pela orientação na análise estatística dos dados.

\section{REFERÊNCIAS BIBLIOGRÁFICAS}

1. Alcantara A, Baruffa G, Aquino Neto JO, Olhinto A, Salvodi T, Lassen C. Epidemiologia da doença de Chagas no RS - Revisão de pacientes após 10 anos de evolução. I - Metodologia. II - Grupo chagásico. III Grupo controle. Anais do XIX Congresso da Sociedade Brasileira de Medicina Tropical, Rio de Janeiro, p 35-37, 1983.

2. Brasil A. A mutabilidade electrocardiográfica na cardiopatia crônica chagásica. Revista da Associação Médica de Minas Gerais 4: 149-152, 1953.

3. Castro $\mathrm{CN}$. Influência da parasitemia no quadro clínico da doença de Chagas. Revista de Patologia Tropical 9: 73-136, 1980.

4. Coura JR, Abreu LL, Pereira JB, Willcox HP. Morbidade da doença de Chagas. IV - Estudo longitudinal de dez anos em Pains e Iguatama, Minas Gerais, Brasil. Memórias do Instituto Oswaldo Cruz 80: 73-80, 1985.

5. Dias JCP. Doença de Chagas em Bambui, Minas Gerais, Brasil. Estudo clínico-epidemiológico a partir da fase aguda, entre 1940 e 1982. Tese de Doutorado, Universidade Federal de Minas Gerais, Belo Horizonte, 1982.

6. Dias JCP, Kloetzel K. The prognostic value of the electrocardiographic features of chronic Chagas' disease. Revista do Instituto de Medicina Tropical de São Paulo 10: 158-162, 1968.

7. Macedo VO. Influência da exposição à reinfecção na evolução da doença de Chagas (estudo longitudinal de 
cinco anos). Revista de Patologia Tropical 5: 33-116, 1976.

8. Maguire JH, Mott KE, Hoff R, Guimarães A, Franca JT, Souza JAA, Ramos NB, Sherlock IA. A three-year follow-up study of infection with Trypanosoma cruzi and electrocardiographic abnormalities in a rural community in Northeast Brazil. The American Journal of Tropical Medicine and Hygiene 31: 42-47, 1982.

9. Moleiro F, Pifano CF, Anselmi A, Ruesta V. La dinámica epidemiológica de la enfermedad de Chagas en el Valle de los Naranjos, Estado Carabobo, Venezuela. Archivos Venezoelanos de Medicina Tropical y Parasitologia Médica V: 47-83, 1973.

10. NYHA (New York Heart Association). Nomenclature and criteria for diagnosis of disease of the heart and great vessels. $7^{\text {th }} \mathrm{Ed}$. Little and Brow Company, Boston, 1973.

11. Pereira JB, Coura JR. Morbidade da doença de Chagas em populações urbanas do Sertāo da Paraiba. Revista da Sociedade Brasileira de Medicina Tropical 20: 101-107, 1987.

12. Pereira JB, Willcox HP, Coura JR. Morbidade da doença de Chagas. III - Estudo longitudinal de seis anos em Virgem da Lapa, MG, Brasil. Memórias do Instituto Oswaldo Cruz 80: 63:71, 1985.

13. Prata A. Prognóstico e complicações da doença de Chagas. Revista Goiana de Medicina 5: 87-96, 1959.

14. Puigbó JJ, Rhode JRN, Barrios HG, Yépez CG. Cuatro años de estudio longitudinal de una comunidad rural con endemicidad chagásica. Boletín de La Oficina Sanitaria Panamericana 66: 112-120, 1969.

15. Sheps MC. An examination of some methods of comparing several rates of proportions. Biometrics 15: 87-97, 1959. 